\title{
César Vallejo oder die Verschmelzung von Leben, Lyrik und Politik
}

Nähern wir uns nun einer weiteren der großen Figuren lateinamerikanischer Avantgarde an, die in ihrem Leben und Werk gemeinsam mit Vicente Huidobro die enorme Breite, aber auch herausragende Qualität der amerikanischen Avantgarden belegt: César Vallejo! Er wurde am 16. März 1892 in Santiago de Chuco in den peruanischen Anden geboren und starb am 15. April 1938 in Paris. Der große peruanische Dichter kam als elftes Kind eines kleinen Verwaltungsbeamten auf die Welt. Seine Großväter waren spanische Priester, die Großmütter Indianerinnen: Vallejo sollte sich dieser Herkunft stets sehr bewusst bleiben und die indigene gemeinsam mit der sozialen Dimension in seinem Schreiben stark machen.

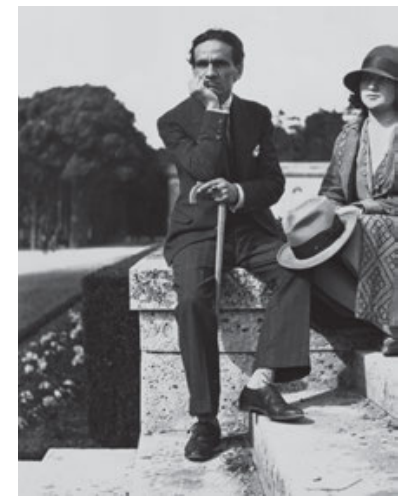

Abb. 63: César Vallejo (Santiago de Chuco, Peru, 1892 Paris, 1938).

Sein ganzes Leben stand im Zeichen finanzieller Schwierigkeiten und Nöte. In seiner Jugend lernte er die rücksichtslose Ausbeutung des indigenen Proletariats in aller Schärfe kennen. Nach Abschluss der Grund- und Oberschule schrieb er sich für ein Medizinstudium in Lima ein, das er aus finanziellen Gründen jedoch niemals wirklich aufnehmen konnte. Ab 1913 erlaubt ihm eine Anstellung als Lehrer die Aufnahme eines Philologie-Studiums an der Universität von Trujillo, das er mit einer Arbeit über die spanische Romantik beendete; 1915 schloss sich ein Studium der Rechtswissenschaften an. Ab 1918 finden wir ihn in der peruanischen Hauptstadt Lima, wo er vorübergehend an einer Privatschule Arbeit fand. Es sind Jahre harter Schicksalsschläge, denn mehrfach erschütterte ihn der Tod ihm nahestehender Personen: 1915 starb sein jüngster Bruder Manuel, 1918 seine Mutter, 1919 der mit ihm befreundete Schriftsteller Abraham Valdelomar. Mitte 1920 ist er in einen obskuren Aufstand verwickelt, in dessen Folge er vier Monate 
ohne jeden Grund inhaftiert wurde. Aus Furcht vor weiteren Verfolgungen entschloss er sich, zusammen mit einem Freund 1923 nach Europa zu reisen. Es war eine gegenüber Vicente Huidobro ganz andere Parisreise: Denn in der französischen Hauptstadt lebte er kümmerlich von wenigen Artikeln und journalistischen Arbeiten für peruanische Periodika sowie von Übersetzungen.

Einen ebenso biographischen wie ideologischen Einschnitt bedeuteten drei Reisen in die damalige Sowjetunion in den Jahren 1928, 1929 und 1931, teilweise finanziert von seiner späteren Frau Georgette. Ende 1930 aber musste er wegen seiner kommunistischen Kontakte Frankreich verlassen und zusammen mit Georgette nach Madrid überwechseln, wo sich seine prekäre finanzielle Lage kaum verbesserte. Anfang 1932 kehrte er heimlich nach Paris zurück, wurde im Sommer aber schon von der Polizei aufgegriffen und verhaftet: Allein seine Zusage, sich aller politischen Aktivitäten zu enthalten, bewahrte ihn vor der Abschiebung. Vergeblich versuchte er, von seinem Schreiben in Paris zu leben; auch seine Theaterversuche fielen durch. Der Spanische Bürgerkrieg elektrisierte ihn; er nahm an der antifaschistischen Solidaritätsbewegung teil, gründete zusammen mit Pablo Neruda 1937 das „Comité Ibero-Americano para la defensa de la República Española“ und figurierte ebenfalls beim Internationalen Kongress von Schriftstellern und Intellektuellen gegen den spanischen Faschismus in Valencia und Madrid. Seine politischen Überzeugungen hatten sich längst gefestigt.

Erst nach seinem frühen Tod im Jahr 1938 - er ist auf dem Cimetière de Montparnasse beerdigt - fand sein gewaltiges dichterisches Schaffen zunehmend Anerkennung. Seine lyrischen Anfänge standen noch im Schatten des Modernismo, insbesondere Rubén Daríos, doch wies sein erster Gedichtband Los heraldos negros von 1919 bereits eine höchst individuelle Ausrichtung auf: Seine lyrische Intensität und Vehemenz, seine bisweilen chaotische Anhäufung von Lexemen und die Überschreitung, ja Sprengung aller Grenzen von Konvention und traditioneller Rhetorik zeichneten bereits seinen ersten Band aus. Dichtung war für Vallejo die Möglichkeit, alle Bereiche des Lebens zu erfassen, Leben in seiner intimsten Form zugänglich zu machen und zugleich in seiner Schönheit und obskuren Unergründlichkeit verwundert und bewundernd zu präsentieren. Vallejos Themen ergaben sich geradezu organisch aus seinem intensiven Erleben aller Dimensionen menschlicher Erfahrung, die assoziativ und a-logisch miteinander verknüpft werden. Wir werden uns gleich mit dem Titelgedicht von Los heraldos negros von César Vallejo beschäftigen.

Bereits 1922, also noch vor seiner Parisreise, legte er mit dem Gedichtband Trilce einen faszinierenden poetisch verdichteten Bruch mit allen Regeln herkömmlicher Sprache vor, der bis über die Grenzen des Unverständlichen und nur mehr Erahnbaren hinausging. Schon der Titel gab Rätsel auf, wurde als Steigerungsform von „dulce“, aber auch als Zusammenschluss von „triste“ und „dulce“ 
gedeutet. Vallejo überforderte bewusst sein zeitgenössisches Publikum mit einer Dichtung, welche der Sprache alles abverlangte und Gewalt antat: Er versuchte, ins Unbewusste der Sprache und zugleich des Lebens vorzudringen und seiner Leserschaft keinerlei Strapazen auf dieser Reise zu ersparen.

Vallejos letzter, 1938 erstmals veröffentlichter Gedichtband España, aparta de mi este cáliz konnte erst postum ediert in den Poemas humanos in Paris am Vorabend des Zweiten Weltkriegs erscheinen und drückte die ganze Erschütterung aus, welche der Spanische Bürgerkrieg in Vallejo ausgelöst hatte. In den Kompositionen dieses Bandes drang Vallejo in einer gegenüber Trilce weniger radikalen Sprachdekonstruktion zum menschlichen Gehalt des Sterbens und des Weiterlebens, zum Kreatürlichen wie Schöpferischen im Menschen vor. An Intensität des Humanen in seiner Zerrüttetheit, Zärtlichkeit und Zerbrechlichkeit sind diese Gedichte schwerlich zu übertreffen. ${ }^{1}$

Mit den Poemas humanos, die kurz vor seinem Tod entstanden, hinterließ César Vallejo ein lyrisches Vermächtnis, das zugleich Ausdruck der Avantgarde und deren Überwindung war. Diese Gedichte berühren in besonderem Maße, da sie am Abgrund des Spanischen Bürgerkrieges, aber auch des bevorstehenden Zweiten Weltkrieges im Zeichen der Leiden alles Menschlichen verfasst wurden. Doch im Angesicht von Sterben und Tod glimmt noch immer eine letzte Hoffnung auf Erlösung des Menschen von all seinen Qualen auf. Den Visionen des Dichters vom eigenen Tod haftet indes etwas Ruhiges, bisweilen fast Heiteres an, so dass der Blick auf das Überindividuelle durch das Individuum selbst stets von neuem geschärft wird.

César Vallejo hat auch Prosa, Kurzromane und unter anderem den Roman Tungsteno 1931 verfasst, in welchem er aus seiner politischen Perspektivik das Elend der Arbeiter und die Knechtschaft der indigenen Bewohner Perus in scharfen Schnitten zum Ausdruck brachte. Seine kommunistischen Überzeugungen waren auch in diesem, auf realen Ereignissen beruhenden Erzähltext in einer tiefen Anschauung des Humanen fundiert. Daneben erfreuen sich seine Erfahrungen in der Sowjetunion (Rusia en 1931) heute eines verstärkten Interesses, ${ }^{2}$ da sie ein entscheidendes Licht auf die Annäherungen zwischen künstlerisch-literarischer und politisch-ideologischer Avantgarde in der ersten Hälfte des 20. Jahr-

1 Vgl. auch Bosshard, Marco Thomas: Die Reterritorialisierung des Menschlichen in den historischen Avantgarden Lateinamerikas. Für ein multipolares Theoriemodell. In: Asholt, Wolfgang (Hg.): Avantgarde und Modernismus. Dezentrierung, Subversion und Transformation im literarischkünstlerischen Feld. Berlin - Boston: Walter de Gruyter 2014, S. 147-168.

2 Vgl. u. a. das César Vallejo gewidmete Kapitel in García Bonillas, Rodrigo: Escritores iberoamericanos en tierra soviética (1917-1957). Phil. Diss. Universität Potsdam 2021. 
hunderts warfen. Doch auch für César Vallejo war nicht nur die schmerzlich aus der Distanz vermisste Heimat Peru, sondern das weltoffene Paris, noch immer die „ville-lumière“ der Lateinamerikaner, der künstlerische Bezugspunkt und zugleich sein Lebenszentrum, obwohl Vallejo im Gegensatz zu Huidobro nicht aus führenden Gesellschaftsschichten, sondern dem einfachen Volk seiner peruanischen Heimat stammte.

Schon der Physiognomie César Vallejos war die indigene Abkunft und die Herkunft aus einem kleinen Dorf in den peruanischen Anden stets zu entnehmen. Er blieb sich dieser Wurzeln seiner Existenz stets bewusst - ein wenig so wie Rubén Darío, der auch als Dichterfürst keineswegs seine indianische Abkunft verschwieg, sondern in manchen Gedichten explizit an diese anknüpfte und sich auf indianische lyrische Traditionen bezog. Schon aus den hier versammelten Biographemen wird jedoch ersichtlich, dass César Vallejo im Gegensatz zu Rubén Darío und Vicente Huidobro weniger auf der Sonnenseite des Lebens stand. Als Dichter genoss er zu Lebzeiten nur bescheidenen Erfolg und machte schließlich auch in seinem politischen Engagement eher traumatische Erfahrungen, die er in España, aparta de mí este cáliz zu höchstem lyrischem Ausdruck erhob.

In einem César Vallejo gewidmeten Abschnitt seiner berühmten Siete ensayos ließ José Carlos Mariátegui nicht ohne Grund mit Los heraldos negros (1918 laut Impressum, eigentlich 1919 erschienen) die neue peruanische Lyrik beginnen, ordnete diesem Gedichtband folglich eine Gründerfunktion innerhalb eines neuen literarischen Peru zu. Bleiben wir einen Augenblick bei Mariáteguis Sichtweise des großen peruanischen Dichters, da sie zeigt, dass Vallejo bei einer kulturellen Elite in Peru durchaus ein wichtiger Orientierungspunkt war, obwohl er - wie Mariátegui betonte - unerkannt durch Limas Straßen gegangen sei! Vallejo, der für Mariátegui der „poeta de una estirpe, de una raza“ ist, bringe zum ersten Mal in der peruanischen Literatur ein „sentimiento indígena virginalmente expresado“, ein geradezu jungfräulich ausgedrücktes indigenes Gefühl zum Ausdruck.

César Vallejo wird zum absoluten Schöpfer, zum Kreator stilisiert, wofür das Titelgedicht von Los heraldos negros, das uns jetzt beschäftigen wird, beispielhaft steht. Für Mariátegui vereinte Vallejo Elemente des Symbolismus, des Expressionismus, des Dadaismus und des „Suprarrealismo“, also des Surrealismus, in seiner verdichteten lyrischen Sprache. Charakteristisch aber sei die „nota india“ in seinem dichterischen Werk, ohne jede Folklore und ohne jede Nostalgie. José Carlos Mariátegui sieht die Lyrik Vallejos sehr wohl als einen Bruch mit der höfischen Lyrik der ,Lakaien‘, die zuvor in seinem Heimatland dominiert habe. Vallejo sei letztlich ein „místico de la pobreza“, ein Mystiker der Armut gewesen: ein wirklicher Schöpfer und authentischer Auctor. Und eben hierin liege seine Einzigartigkeit und seine Größe in der Literatur. 
Wie kein anderer, so Américo Ferrari, habe César Vallejo in den zwanziger und dreißiger Jahren die Freiheit der dichterischen Sprache verkörpert. ${ }^{3}$ Im Bereich der Lyrik tauchte Vallejo ja nur wenige Jahre nach Vicente Huidobro auf und signalisierte zusammen mit diesem eine grundsätzliche Neuorientierung der hispanoamerikanischen Lyrik am Experimentellen, am Radikalen, stand für eine Tendenz zum Überschreiten bislang eingehaltener Grenzen des Logischen, des Kausalen. Dabei schrieb César Vallejo eine im Gegensatz zu Vicente Huidobro weitaus stärker im andinen Raum verwurzelte und auf dessen kulturelle Traditionen aus der Distanz bezogene Lyrik, die überdies eine klare ideologische Leitlinie besaß, welche ihn mit der politischen Avantgarde seiner Zeit verband.

Vallejos erster, im Juli 1919 ausgelieferter Gedichtband Los heraldos negros ist ein in mehrfacher Weise hybrides Werk, zeigen die verschiedenen hier vereinigten Tendenzen doch unverkennbar - wie schon angedeutet - zum Teil noch auf den Modernismo eines Rubén Darío oder eines Julio Herrera y Reissig sowie auf den Symbolismus europäischer Provenienz. Auf der anderen Seite findet sich in ihnen ein neuer, am Abrupten, am Suchenden, am Fragenden und am Radikalen orientierter Ton, der die absolute Neuheit dieses Bandes ausmacht. Diese Töne, die eine bisweilen eher noch konventionelle Lyrik durchschlagen, sind deutlich vernehmbar in Vallejos Titelgedicht, das ich Ihnen in der mitunter zwar nicht unproblematischen, aber kongenialen Übersetzung von Hans Magnus Enzensberger präsentieren möchte:

Es gibt Schläge im Leben, so hart ... Ich begreife es nicht!

Als schlüge Gott zu in seinem Hass; als trieben sie der Seele die Brandung alles Erlittenen zu und stauten sie auf in ihr ... Ich begreife es nicht!

Sie kommen nicht oft, doch sie kommen ... Und reißen finstere Gräben ins wildeste Antlitz und in die kräftigsten Lenden.

Wie die Hengste barbarischer Hunnenfürsten oder die schwarzen Boten, die der Tod nach uns ausschickt.

Die tiefen Abstürze der Gekreuzigten unserer Seele, eines göttlichen Glaubens den das Verhängnis lästert.

Blutige Schläge: ein anderes Brot platzt auf unter ihnen, ein Brot das uns verbrennt an der Ofentür.

3 Vgl. Ferrari, Américo: César Vallejo entre la angustia y la esperanza. In: Vallejo, César: Obra poética completa. Madrid: Alianza Editorial ${ }^{2} 1983$, S. 9-55. 


\begin{abstract}
Und der Mensch ist elend ... elend! Er wendet die Augen, es trifft ihn wie ein Handschlag auf den Rücken;

Er wendet die irren Augen, und alles Erlebte staut sich, wie eine Pfütze von Schuld, in seinem Blick.
\end{abstract}

Es gibt Schläge im Leben, so hart ... Ich begreife es nicht! ${ }^{4}$

Wir erkennen auf den ersten Blick, dass wir es in diesem Titelgedicht mit einer völlig anderen Lyrik zu tun haben, der das spielerische Element weitgehend abhandengekommen ist. Es geht ganz allgemein in der Lyrik Vallejos um die Conditio humana, um das Existenzielle, ja mehr noch: um das Elend des Menschen. Natürlich war uns das Spiel mit Leben und Tod, mit dem ständigen Vergehen, dem Weißwerden der Haare, dem Leben und Sterben durch die allegorische Veranschaulichung der Windmühlenflügel in Vicente Huidobros Bildgedicht Moulin symbolhaft vor Augen geführt worden. Und doch lag etwas Spielerisches, Leichtes und um die ästhetischen Ausdrucksformen Kreisendes in diesem Gedicht gewesen, das gerade einmal zwei Jahre nach dem uns hier interessierenden Gedicht César Vallejos veröffentlicht wurde.

In Los heraldos negros jedoch trifft uns die existentielle Dimension mit aller Wucht. Ich habe bereits betont, dass Vallejo durch den Verlust von Freunden und Familienangehörigen, vor allem aber seiner Mutter tief getroffen wurde: Es waren starke Schläge, denen der junge Dichter allein seine Lyrik entgegenzusetzen vermochte. Freilich nicht im Sinne einer erklärenden oder gar verklärenden Antwort, sondern als ein verzweifeltes „Yo no sé“, als ein obsessives „Ich-weiß-nicht“, das seine Lyrik und sein Leben ständig miteinander verbinden und durchziehen sollte. Der Mensch erscheint in diesem Gedicht als „pobre ... pobre“ im Fadenkreuz ihn übersteigender Gewalten, als Opfer göttlichen Hasses, Gegenstand und Gehandelter der schwarzen Boten des Todes, als vom Schicksal im negativen Sinne Auserkorener, auf Du und Du mit dem Tod, dem göttlichen Hass, jenen Boten, die ihm gleichsam von hinten, unerwartet, auf die Schulter klopfen.

Bei César Vallejo hat die Lyrik einen anderen ,Sitz im Leben': Sie wird stets zum Experimentellen, zur absoluten Grenze des Denkens und mehr noch des Sprechens vorwärtsgestoßen und doch stets rückgekoppelt bleiben an die existentielle Grunderfahrung des Ausgeliefertseins menschlichen Daseins. Lyrik wird zur verdichtetsten Form menschlichen Lebenswissens und menschlichen Erle-

4 Vallejo, César: Die schwarzen Boten [Übersetzt von Hans Magnus Enzensberger]. In: Köhler, Hartmut (Hg.): Poesie der Welt: Lateinamerika. Berlin: Edition Stichnote im Propyläen Verlag 1986, S. 151. 
benswissens, zeichnet das auf, was sich - „Yo no sé!“ - jeglicher Rationalität entzieht und doch immer noch in Sprache ausgedrückt werden kann. Denn gerade weil der Mensch dem unergründlichen und harten Schicksal nichts Machtvolles entgegensetzen kann, verfügt er doch noch immer über die Sprache, die seinem Elend, seiner Conditio humana, zumindest Ausdruck verleihen mag. War das „Je ne sais quoi“ während langer Jahrhunderte die literarische Formel für das nicht mehr sprachlich Erfassbare, für das Irrationale etwa in der Schönheit, ${ }^{5}$ so werden die Hammerschläge des „Yo no sé“ nun zum fast geschrienen Ausdruck menschlichen Elends.

Die literarische, poetische Übersetzung durch den deutschen Dichter und Essayisten Hans Magnus Enzensberger, einen der großen Vermittler lateinamerikanischer Literatur im deutschsprachigen Raum, zeigt uns nicht nur, dass literarische Übersetzungen von großem Wert gerade auch für die Untersuchung semantischer Aufladungen und Polyvalenzen sein können. Darüber hinaus findet sie Äquivalente für die ausdrucksstarke Dichtkunst dieses jungen Peruaners, der sich zum damaligen Zeitpunkt noch in Peru, weitab von der literarischen Szene Europas, aufhielt.

Bei Durchsicht der Verse Enzensbergers erkennen wir sofort, dass die Akzente seiner Übersetzung bereits im Ausgangs- und Endvers des Gedichts anders gesetzt sind. Die erste und letzte Gedichtzeile sind identisch beziehungsweise unterscheiden sich nur darin, dass beim ersten Auftauchen der erste Vers in eine Strophe integriert, beim letzten Vers aber dann als Solitär dasteht, was von größter Bedeutung für die Semantik des gesamten Gedichts ist. Denn es deutet sich keine Lösung, keine Entwicklung an: Der Mensch ist gefangen, abhängig, ausgeliefert allen Gewalten und dem göttlichen Hass. Reicht da das von Enzensberger vielleicht auch aus rhythmischen Gründen gewählte Lexem „begreifen“?

Meine Antwort auf diese Frage lautet: Ich weiß nicht! Mir scheint, dass die logische Konnotation des Begreifens zu stark ist, um das A-Logische und die existentielle Betroffenheit in ihrem breiten Bedeutungsfächer auszudrücken, eine Betroffenheit, die keineswegs nur logisch-rationaler Natur ist, sondern ganz existenziell alle auch irrationalen Bereiche des Menschen erfasst. Das rationale Wissen in all seiner Breite ist hier nicht mehr fähig, dem Tod, dem blind zuschlagenden Hass, ja der unverschuldet erworbenen Schuld auf die Spur zu kommen und Ausdruck zu geben. So kommt denn Wahnsinn auf in den Augen des betroffenen, des getroffenen Menschen. Der „hombre“ ist „pobre“, ein Echo, das die

5 Zur Bedeutung des „Je ne sais quoi“ vgl. die erhellende Studie von Köhler, Erich: „Je ne sais quoi“. Ein Kapitel aus der Begriffsgeschichte des Unbegreiflichen. In (ders.): Esprit und arkadische Freiheit. Aufsätze aus der Welt der Romania. Frankfurt am Main: Klostermann 1966, S. 230 ff. 
Silben des Wortes „Mensch“ widerhallen lässt: Vallejo spielt mit dem Echo des Lexems „Mensch“ im Armen, im Elenden.

An dieser Stelle scheint mir die Übersetzung von Enzensberger auch gut gewählt, denn der Mensch ist eben elend, elend: Auch hier ist der Vokal „e“ beibehalten. Die Pluralbildung „Cristos“, die im Deutschen nicht als „Christen“ und schon gar nicht als „Christusse“ übersetzbar wäre, zeigt uns die existentielle Dimension der religiösen Symbolik des Gedichts auf, die keineswegs sakral, sondern stark desakralisiert anklagend verwendet wird. Der biblische Vergleich des Menschen mit dem Brot, das gegen Ende des Gedichts nahe der Ofentür verbrennt, ist eine weitere Symbolik christlicher Provenienz, die zudem die ganze Absurdität zum Ausdruck bringt, der zufolge ein Lebens-Mittel zum Aufplatzen und zum Verbrennen, so nahe an seiner Vollendung und Erlösung, gebracht wird.

Wir könnten in diesem Vergleich nicht nur eine Reminiszenz an die Bibel, sondern auch und gerade an indigene Mythen und Vorstellungen sehen, denen César Vallejo immer, auch in seinem späteren Leben in Europa, sehr nahe war. Wusste der in den peruanischen Anden geborene Dichter um den indianischen Mythos vom gebackenen Brot, dem Schöpfungsmythos des Menschen, bei dem Gott drei Brote in den Ofen stellt? Das erste nimmt er zu früh heraus, es bleibt ein wenig weiß noch, das zweite ist gerade rechtzeitig dem Ofen entnommen, es ist knusprig braun, doch über dieser Freude vergisst Gott das letzte Brot, das er zu spät aus dem Ofen herausholt, ist es nun doch schwarz geworden. Die Präsenz der mythischen Verbindung von Brot und Mensch - hier im Triptychon der Genesis der weißen, der indigenen und der schwarzen Menschen - ist allgegenwärtig und in dieses Gedicht eingewoben. So lassen sich selbst in Los heraldos negros die Spuren indigener Mythen und Mythologien finden, mit denen César Vallejo stets sehr kreativ arbeitete und damit eine Traditionslinie der lateinamerikanischen Avantgarden schuf, in die sich in Mexiko auch ein Alfonso Reyes einschreiben konnte.

In einem christlichen Traditionszusammenhang bewegt sich wiederum die Rede von der Seele, in welcher sich das Erlittene, das menschliche Leiden, anstaut und nur durch die Augen, einem alten Topos europäischer und speziell spanischer Mystik zufolge die Fenster der Seele, nach außen dringt: als Wahnsinn, der vom Menschen im Menschen nur mühsam zurückgehalten werden kann. Die Conditio humana ist unverkennbar die des Leidens, für die Christus als Gekreuzigter, nicht aber als Erlöser steht. Denn eine Erlösung ist nicht in Sicht, Überall schwärmen die schwarzen Herolde, die Heraldos negros, aus und bedrohen ein günstiges, gnädiges Schicksal des Menschen.

Ist ihr Ruf der des Todes, des Hasses oder der eines Gottes? Oder ist es genau die Verknüpfung dieser Ruf des göttlichen Hasses, von der im Gedicht so dunkel die Rede ist? 
Der Dichter, das lyrische Ich, findet hierauf keine Antwort. Die groß geschriebenen Lexeme der einzelnen Strophen zeigen dies bereits an: in der ersten Strophe „Dios“, in der zweiten ganz am Ende „Muerte“, in der dritten schließlich „Cristos“ und vor allem „Destino“, das erneut in einer Art Echowirkung zu „Cristos“ steht. Nach Gott, dem Tod, „Cristos“ und dem Schicksal aber steht in der vierten Strophe der Mensch allein - und zwar nicht als groß geschriebener „Hombre“, sondern gerade als Armer, als Elender. Er ist den Schlägen schutzlos ausgeliefert, die ein Mächtigerer ihm versetzt, als es selbst der stärkste und stolzeste (und nicht so sehr der „wildeste“, wie Hans Magnus Enzensberger übersetzt) Mensch sein könnte. Was kann hier selbst noch von der „fe adorable“, vom bewundernswertesten Glauben, übrigbleiben?

Nicht nur die logisch-kausale, sondern auch die mythisch-religiöse Tröstung bleibt dem Menschen verwehrt: Das kausale Prinzip ist völlig außer Kraft gesetzt, denn die Schuld ist bereits in der bloßen Existenz des Menschen zu finden. Daher sollte man „fiero“ nicht mit „wild“, sondern eher mit „stolz“ übersetzen: Nicht ein Wilder wird hier gezähmt, sondern ein selbstbewusster, stolzer Mensch gedemütigt. Zugleich erinnern die tiefen Gräben, welche hier in das Antlitz dieses Menschen gerissen werden, exakt an die Metaphorik Shakespeares in einem seiner berühmtesten Sonette, When forty winters. Dort sind es freilich die vierzig Winter, also das unvermeidliche Altern, die die Risse und Gräben in ein ehedem schönes Antlitz gezogen haben, und nicht die Schläge der schwarzen Boten.

Es wäre sehr wohl möglich, die „pobreza“ des Menschen, die Armseligkeit, seine Armut, sozial zu lesen und mit der Solidarität César Vallejos mit den Armen, den Entrechteten und seiner politischen Parteinahme für sozialistische und kommunistische Ideen in Verbindung zu bringen. Es ist die Verteidigung, des Kreatürlichen, der Benachteiligten, der den Schlägen von oben Ausgesetzten, die über keine Möglichkeit verfügen, den „heraldos negros“, den schwarzen Boten gleich welcher barbarischen Macht, etwas entgegenzusetzen. Wir konstatieren, dass sich auch in diesem Gedicht die Polysemie nicht als ein freies Spiel des Signifikanten mit der „signifiance“ und damit als ein beliebiges Spiel der Signifikanten untereinander zu erkennen gibt. Dies gilt es angesichts aller Experimentierfreudigkeit mit dem Wortmaterial, wie sie nicht zuletzt die späteren Gedichte Vallejos in verstärktem Maße aufweist, nicht aus den Augen zu verlieren. Die unterschiedlichen Isotopien oder Bedeutungsebenen Vallejos sind immer an die existentielle Dimension des Menschen rückgebunden und verweigern sich jeglichem reinen Spielcharakter.

Vor diesem Hintergrund darf man Américo Ferrari Recht geben, wenn dieser betont, die Lyrik in César Vallejos 1922 erschienenem Band Trilce sei in diesem Sinne nicht experimental, sondern ,experiencial“. Sie verfügt damit noch immer über eine Grundlage an Erfahrungen und an Lebenswissen, was im Übrigen auch 
durch Briefe Vallejos aus jenen Jahren bestätigt wird. Vallejos Lyrik ist in diesem Gedichtband radikal, eine Absage an modernistische Traditionen und ein konventionelles Gedichtverständnis, eine nach den Wurzeln des Lyrischen im Eigenen suchende Haltung, die - so könnten wir vielleicht am besten formulieren - das Experimentelle mit dem Existentiellen und Erfahrenen in einem poetisch verdichteten Lebenswissen zu verbinden sucht.

Bereits der Titel ist Programm: Trilce suchen Sie vergeblich in einem Wörterbuch der spanischen Sprache. Es ist - wie schon betont - möglicherweise eine Vermischung von „triste“ und „dulce“, vielleicht aber auch eine Bildung wie „triple“ am Gegenstand des „dulce“. Vallejo lässt dies offen. Wie dem auch sei: Hier nimmt sich der peruanische Dichter in radikaler Weise seine dichterische Freiheit und provoziert sein Lesepublikum.

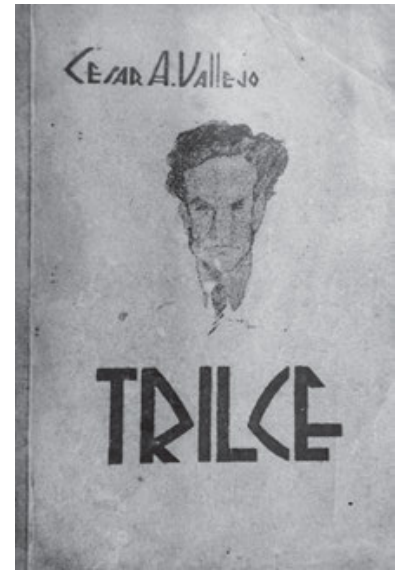

Abb. 64: Cover der Erstausgabe von César Vallejos Trilce, 1922.

Ich wiederhole es gerne: Vallejos Lyrik ist kein freies Spiel der Kunst und auch kein Glasperlenspiel! Den existentiellen Hintergrund dieses Gedichtbandes bilden die Verarbeitung des Todes der Mutter, die unschuldige Inhaftierung für mehr als vier Monate, ein Gefühl der radikalen Einsamkeit und Verwaisung, wie es sich in diesen Gedichten wiederfinden lässt. Zugleich und vor allem aber ist es eine konsequente Suche nach Freiheit, welche der Band offeriert. Wenn er es jetzt nicht versuche, so schrieb Vallejo damals an einen Freund, werde er nie frei sein: Das Ergebnis ist Trilce.

Glücklicherweise wählte César Vallejo für seinen Gedichtband nicht den ursprünglich geplanten Titel Cráneos de bronce und - mehr noch - brachten ihn gutmeinende Freunde in letzter Minute davon ab, seine Gedichte unter dem etwas anmaßenden Pseudonym César Perú zu veröffentlichen. Dieses Pseudonym zeigt andererseits aber sehr wohl, in welchem Kontext sich die Lyrik des peruanischen 
Dichters situierte und welche nationale Repräsentativität er sich selbst zuschrieb. Denn aufgewachsen in einem kleinen Andendorf in mehr als dreitausend Meter Höhe verstand er sich als leidender Ausdruck seines gequälten und ausgebeuteten Heimatlandes Peru.

Die insgesamt siebenundsiebzig Gedichte, die keine Titel tragen, sondern römisch durchnummeriert sind, entstanden teilweise aus vorangehenden Gedichten, sind also auf eine intensive intratextuelle Arbeit und einen starken Umprägungswillen, einen Stil-Willen zur Veränderung, geprägt. Wie bei César Vallejos Lyrik üblich, finden sich auch in diesem Band autobiographische Reminiszenzen, Erinnerungen an die Kindheit, an gescheiterte Liebesbeziehungen und das Leiden der indigenen Bevölkerung, so dass trotz aller provokativen äußeren Gestalt eine existentielle Linie aufrechterhalten bleibt. Dies hindert César Vallejo nicht daran, Gedichte an die Grenze zum Nicht-Sagbaren, ja zum Nicht-mehr-Verstehbaren vorzuschicken, ja die Grenze zur Inkohärenz zu überschreiten. Nehmen wir als Beispiel Gedicht XII:

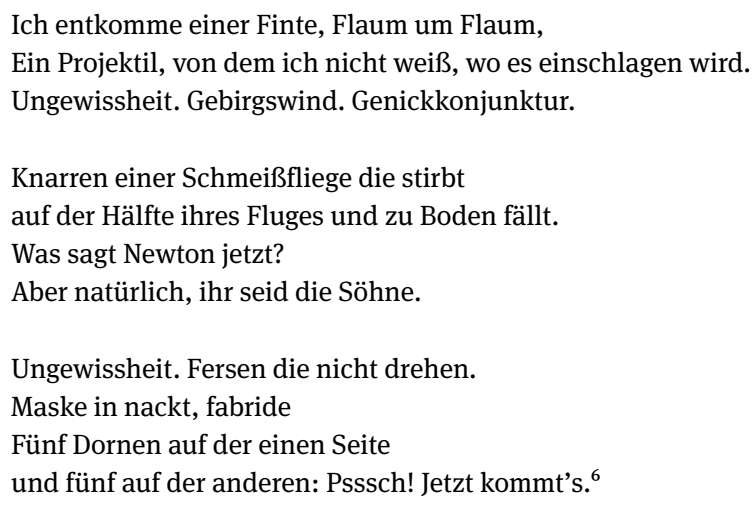

Das Gedicht erinnert einen an die bisweilen unbeholfenen Deutschstunden und an die klassische Frage: Was will der Dichter uns hiermit sagen? Auf den ersten Blick scheinen die Inkohärenz des Gedichts vollständig und der Sinn obskur. Doch versuchen wir es einmal mit dieser lyrischen Struktur, die sich - ganz im Sinne der negativen Ästhetik eines Theodor W. Adorno - der leichten Konsumierbarkeit durch breite Leserschichten entzieht! Dieses Gedicht setzt die eigene Lesegeschwindigkeit radikal herab und zwingt einen, jeden Vers und jedes Lexem ganz genau durchzusehen und Relationen innerhalb der Gedichtstruktur herzustellen.

6 Vallejo, César: Trilce. Lima: Ediciones Laberintos / Centro Peruano de Estudios Culturales 2008, S. 19. 
Immerhin gibt es bestimmte Merkmale und Besonderheiten, auf die wir achten sollten. Da sind zum einen die Lexemrekurrenzen wie etwa „caer“ oder vor allem ,incertidumbre“, das gleich zweimal alleine steht und damit in eine besonders wichtige Position einrückt. Die „,incertidumbre“, die Ungewissheit, lässt sich in der Tat auch auf zwei Ebenen verankern: Zum einen erfolgt dies auf der Ebene einer Selbstreferentialität des Gedichts in Bezug auf die Aneignung durch die Leserschaft, die mit einem höchsten Gefühl an Unsicherheit in diesem Gedicht konfrontiert wird. Die Ungewissheit bezieht sich somit auf den Kodierungs- und Dekodierungsprozess, insofern eine hohe Ungewissheit besteht, wie die Lexeme des Gedichts und dessen gesamte Struktur zu deuten sind und wie die - um mit Wolfgang Iser zu sprechen - Unbestimmtheitsstellen des Gedichts semantisch aufgefüllt werden können. Es geht also darum, diese Unbestimmtheiten mit verschiedenen Deutungsansätzen zu füllen und damit für eine grundlegende Polysemie des Gedichts zu optieren.

Zum anderen sollten wir unseren Blick aber auch auf die referentielle Ebene richten, denn die Situation der Ungewissheit und Unsicherheit charakterisiert auch die semantischen, rhythmischen oder klanglichen Vorgänge und Verfahren innerhalb des Gedichts selbst. Was geschieht hier? Wir haben es mit einer dreizeiligen und zwei vierzeiligen Strukturen zu tun, was nach Adam Riese 11 Verse macht. Bei einer ungeraden Vers-Zahl schauen wir uns immer am besten gleich mal die Zentralachse an, in diesem Falle also den sechsten Vers! Und der ist eine Frage: „¿Qué dice ahora Newton?“

Isaac Newton steht innerhalb der abendländischen Tradition gleichsam als Emblem für die Wissenschaftlichkeit, für die Verknüpfung von Ursache und Wirkung, für eine naturwissenschaftlich begründete Logik, die wir mit Jacques Derrida als logozentrisch charakterisieren könnten. Das Zur-Erde-Fallen der im Fluge verstorbenen Fliege spielt an auf die berühmte Episode, in welcher Newton die Erleuchtung hinsichtlich der Schwerkraft durch einen auf den Boden fallenden Apfel kam. Fragt sich nur noch, was Newton hier sagen würde oder gesagt hätte. Denn gewiss entspricht das „caer“, das Zu-Boden-Fallen der Fliege allen Gesetzen und Berechnungen der Gravitationskraft durch den Briten.

Im nachfolgenden Vers wird „natürlich“ („naturalmente“) eine Linie gezogen zu einem „vosotros“, wobei die angesprochenen Personen dieser zweiten Person Plural „natürlich“ Söhne oder Kinder („hijos“) seien. Söhne Newtons vielleicht, also Newtonianer? Und damit Anhänger einer Rationalität, die auf Logik, Ableitbarkeit und Kausalität setzt. Oder sind es Söhne einer Mutter, die Vallejo gerade zu jener Zeit verloren hatte? Die Antwort gleich zu Beginn des letzten Verses: „Incertidumbre“. Die in der Schwebe gehaltene Unsicherheit und Ungewissheit erschien bereits im zweiten Vers, als es um den fraglichen Einschlagsort des Projektils ging, von dem das lyrische Ich nicht $\mathrm{zu}$ berechnen vermochte, wohin es 
fallen (,caer“), wo es also einschlagen werde. Aber wäre der Flug dieses Projektils nicht auch wie der Flug und Fall der Fliege leicht schon vorab zu berechnen? Mag sein, aber nur für die Anhänger Newtons, für Menschen also, die an die (naturwissenschaftliche) Logik glauben. Trifft das Projektil ins Genick? Werden wir Zeugen einer Hinrichtung? Und am Ende des Gedichts: Bezieht sich das „Ya sale“ nicht auf das Projektil? Alles ist ungewiss, aber alles ist zugleich bedrohlich in dieser Finte, aus der das Ich wohl noch einmal entfliehen kann.

Die ,incertidumbre“ findet vielleicht ihren Ausgang am Ende des Gedichts, signalisiert bereits durch die Interpunktion, die konventionsgemäß eine Zusammenfassung oder logische Folge anzeigen müßte: „Chit! Ya sale.“ Ein Geräusch und dann ein Herauskommen. Aber wer oder was kommt hier heraus? Ist es das Geräusch einer sich öffnenden Tür, der Laut eines sich bewegenden Rocks oder eben doch das Projektil? Am Ende dieser „Cervical coyuntura“ steht ein Erscheinen: Vielleicht ist es das Erscheinen der Geliebten des männlichen Ich, das noch mit Blick auf den Flaum sehr jung ist? Ein Rendez-vous mit dem lyrischen Ich, das sich aus Unsicherheit auf seinen Fersen nicht umdreht? Es ist nicht mit Bestimmtheit zu sagen.

Die Ungewissheit ist im Übrigen - auf der Ebene des Protagonisten beziehungsweise des lyrischen Ich - mit konkreter Bedrohung verbunden: Nicht allein der plötzlich abbrechende Flug der Schmeißfliege, sondern mehr noch das Projektil, von dem wir weder wissen, woher es kommt noch was es treffen wird, verheißen nichts Gutes. Auch die Dornen in der letzten Strophe dieses Gedichts aus Trilce verweisen auf ein Leiden, das in einen christlichen, biblischen Zusammenhang gebracht wird, erinnern die Dornen doch an die Dornenkrone Christi. Unklar bleibt, welcher Finte das lyrische Ich entgangen ist. Die Präsenz des Todes zieht sich quer durch das Gedicht und manifestiert sich erstmals in jener Fliege, die zu Boden fällt, einfach so: ohne jeden Grund, ohne jede Kausalität. Was würde Newton dazu sagen?

Es ist augenfällig, dass im naturwissenschaftlichen Bereich gerade in jenen Jahren die Gültigkeit der Gesetze Newtons deutlich beschränkt und andere Logiken in die Naturwissenschaften eingeführt wurden, die mit Einstein und Heisenberg sich neue Denkräume und Logiken eröffneten, welche mit den Anschauungen Newtons nicht mehr zu erschließen waren. Newton wurde damit nicht aus der Welt geschafft, aber doch die Gültigkeit seiner Gesetzmäßigkeiten erheblich begrenzt. Mit anderen Worten: Deutet die verdoppelte ,incertidumbre“ bereits jene wenige Jahre später gefundene Heisenberg'sche Unschärferelation oder Unbestimmtheitsrelation an, welche gemeinsam mit den Erkenntnissen Einsteins die Tür zu den Entwicklungen des 20. Jahrhunderts weit aufstoßen sollten? In jedem Falle verweist das Gedicht auf einen weiten Bereich, der nicht länger den Gesetzen Isaac Newtons und damit einer einfachen rationalen Logik unterworfen ist. 
Zugleich umgeben die einzelnen Elemente der Angst, der Bedrohung und des Todes das Warten und die mögliche Begegnung, die sehr wohl Begegnung mit einer Frau, einer Geliebten sein könnte. Wir finden die Rekurrenz bestimmter verknüpfter Isotopien häufig bei Vallejo, wobei die Verknüpfung von Sexualität und Tod beziehungsweise Bedrohung eine der dauerhaftesten in seinem lyrischen Schaffen ist. Angst und Bedrohung erscheinen nicht als logisch verortet: Wir wissen nicht, ja ahnen nicht einmal wirklich, wovon und von wem die Bedrohung ausgeht! Wir wissen nur, dass es ein Leiden gibt, wissen nur, dass es den plötzlichen Tod gibt, wissen nur, dass es Projektile gibt, die dem Ich als unberechenbar erscheinen.

Die Grundlosigkeit dieser Bedrohung, das schuldlose Verstrickt-Sein in Schuld, ist spätestens seit seiner mehrmonatigen grundlosen Inhaftierung ein Grundthema Vallejos, wie wir schon bei seinen Schwarzen Boten deutlich sehen konnten. Was kann der Mensch, der elend ist, all dem entgegensetzen? Höchstens seinen Schrei, sein Wort, seinen poetisch verdichteten Text. Die A-Kausalität der Bedrohung und der stets vektoriell auf die Zukunft gerichteten Angst ${ }^{7}$ unterstreichen aber nur umso mehr deren Allpräsenz, die dem Gedicht die innere Spannung vermittelt. Hier wird die „incertidumbre“ produktiv: Denn dem Gedicht wohnt eine unbestimmbare Angst vor etwas noch Unbekannten und Ungenannten inne, das sich schon in naher Zukunft ereignen könnte. Es ist die Angst im Angesicht des Todes.

Vielleicht sollten wir das Gedicht zusätzlich im Kontext oder besser noch in seinem Kotext innerhalb von Trilce lesen. Dafür empfiehlt sich zum Beispiel das dreizehnte, also das direkt nachfolgende Gedicht:

Ich denke an Dein Geschlecht.

Einfacher das Herz, denke ich an Dein Geschlecht, angesichts des reifen Söhnens des Tages.

Ich betaste die Knospe des Glückes, rechtzeitig gereift.

Und es stirbt ein altes Gefühl,

degeneriert zu Gehirn.

Ich denke an Dein Geschlecht, fruchtbarere Furche und harmonischer als der Bauch des Schattens, obwohl der Tod empfängt und gebiert von Gott selbst.

7 Vgl. zur Ökonomie und Vektorizität der Angst Ette, Ottmar: Angst und Katastrophe / Angst vor Katastrophen. Zur Ökonomie der Angst im Angesicht des Todes. In: Ette, Ottmar / Kasper, Judith (Hg.): Unfälle der Sprache. Literarische und philologische Erkundungen der Katastrophe. Wien Berlin: Verlag Turia + Kant 2014, S. 233-270. 


\begin{abstract}
Oh Bewusstsein,
denke ich, ja, im freien Vieh,

das Lust empfindet wo es will, wo es kann.

Oh, Skandal voller Honig der Dämmerungen.

Oh stummer Donner.
\end{abstract}

Orennodremmuts! ${ }^{8}$

So finden wir im unmittelbaren Anschluss an die „incertidumbre“ des zwölften Gedichts von Trilce jene Mischung aus „triste“ und „dulce“, welche die Liebeslyrik César Vallejos charakterisiert und vielleicht auch auf den Titel des gesamten Bandes bezogen werden darf. Dem Gedicht liegt eine eindeutige Entwicklung zugrunde, die im dreimaligen Schluss-„,Oh“ kulminiert, nachdem in der zweiten Strophe („Oh Conciencia“) das Gewissen oder - wie wir sagen könnten - das Freud'sche Über-Ich verabschiedet wurde. Das Denken ist in diesem Gedicht keine rationale Tätigkeit. Denn alles Rationale degeneriert, so wie das Geschlecht zum Gehirn, der „sexo“ zum „seso“ degeneriert. Die Minimaldifferenz zwischen diesen beiden Lexemen ist eine, in der die Schärfe und Kraft des „x“ zu einem wesentlich schwächeren „s“ verkommen ist. So bilden Geschlecht und Gehirn die beiden Pole, zwischen denen sich die entscheidenden Bedeutungsebenen in ihren Transformationen entfalten.

Zweifellos ist das „sexo“ in diesem Gedicht zugleich Sex und Gender, biologisch-physisches Geschlecht und kulturelle Geschlechteridentität oder -zuschreibung, letzteres sicherlich vor allem zu Beginn der zweiten Strophe. Doch die orgiastische Grundstruktur des Gedichts deutet darauf hin, dass wir es hier vor allem mit den primären Geschlechtsmerkmalen zu tun haben, welche in ausreichender Klarheit apostrophiert werden. Das Gedicht bleibt diesbezüglich in keiner Weise obskur. Nicht umsonst war der Verabschiedung des Bewusstseins die Verabschiedung des Intellekts und der Ratio vorausgegangen, jenes Lexems nämlich, das hier in gewisser Weise als Degenerierung von „sexo“ erscheint. Der männliche Blick auf das weibliche Geschlecht assoziiert dieses mit der Ackerfurche und der Fruchtbarkeit, ist in diesem Sinne also topisch, wobei die Harmonie der gesamten irdischen und kosmischen Ordnung beschworen wird. Doch selbst hier, beim Vorgang der Empfängnis und der Geburt, ist der Tod im Zeichen des Göttlichen allgegenwärtig. Es ist eine Lust, die stets im Angesicht des eigenen Todes steht. ${ }^{9}$

8 Vallejo, César: Trilce, S. 20.

9 Auf diesen Zusammenhang hat ausführlich unsere Vorlesung LiebeLesen aufmerksam gemacht; vgl. Ette, Ottmar: LiebeLesen. Potsdamer Vorlesungen über ein großes Gefühl und dessen Aneignung. Berlin - Boston: Verlag Walter de Gruyter 2020. 
Nur die Tierwelt scheint „libre“, scheint frei zu sein von den Einschränkungen des Menschen durch Gewissen und Gehirn, durch Bewusstsein und Bewusstwerdung: Allein die Tiere finden und empfinden die Lust dort, wo sie sie finden, ohne Reue.

Für die Menschen freilich ist die freie geschlechtliche, körperliche Liebe noch immer ein Skandal, der bitteren Anstoß provoziert, obwohl er süßen Honig transportiert. In der Unverblümtheit der Sprache besteht das Skandalon des Gedichts selbst: der avantgardistische Bruch mit den Konventionen der bürgerlich geglätteten Darstellung der Frau. Allerdings bricht dieses avantgardistische Poem nicht mit der Sichtweise des männlichen Blickes auf den nackten weiblichen Körper, der hier in seiner vermeintlichen Freiheit zwar zelebriert wird, zugleich aber sehr wohl den gängigen Konventionen männlicher Objektivierung des Frauenkörpers gehorcht.

Diese Vergegenständlichung der Frau und weiblicher Nacktheit ist ebenso patriarchalisch geprägt wie die Assoziierung des weiblichen Geschlechts mit der fruchtbaren Ackerfurche, die sich für den Samen öffnet. Wir finden diesen männlichen Blick der Avantgardisten auf den nackten weiblichen Körper ebenso in Ramón Gómez de la Sernas Band Senos ${ }^{10}$ aus dem Jahr 1918 wie in Louis Aragons Band Le con d'Irène von 1928: Der avantgardistische Bruch der damaligen bürgerlichen Konvention besteht allein in der direkten, unverblümten sprachlichen Adressierung primärer weiblicher Geschlechtsmerkmale. Keinesfalls aber mündet er in eine gegenüber traditionellen Vorstellungen veränderten Rolle und Funktion des weiblichen Gegenübers.

Die Geschlechtlichkeit der Frau wird zugleich aber auch mit „sombra“ und „muerte“, mit jenem Schattenreich assoziiert, das hier fast unübersetzbar weiblichen Geschlechts ist: es ist ,die“ Schatten und ,die‘ Tod, eine ins Weibliche gesetzte Welt. Gegen den Tod, gegen den Schatten wird das „gozar“ gesetzt, das von der Ratio, vom Bewusstsein nicht kontrolliert sein will. Diese Formulierung ist ähnlich und doch zugleich freier als das „brutal deseo“ des Mannes im zuvor untersuchten Gedicht Vicente Huidobros, womit wir erneut daran erinnert werden, wie stark dieses erotische Thema bei den historischen Avantgarden ebenso in Europa wie in Lateinamerika ist. Und wie stark auch dessen patriarchalische Deutung alles überlagert.

Das lyrische Ich in César Vallejos Gedicht will jedenfalls seine Lust nicht länger vom Bewusstsein kontrolliert, sondern vielmehr sofort erfüllt sehen. „We

10 Vgl. hierzu Ette, Ottmar: Mit Haut und Haar? Körperliches und Leibhaftiges bei Ramón Gómez de la Serna, Luisa Futoransky und Juan Manuel de Prada. In: Romanistische Zeitschrift für Literaturgeschichte / Cahiers d'Histoire des Littératures Romanes (Heidelberg) XXV, 3-4 (2001), S. 429-465. 
want it all and we want it now!“ - so ließe sich dies wohl spätachtundsechzigerhaft mit The Doors kommentieren. In jedem Falle handelt es sich keineswegs um eine krepuskuläre Lyrik, wie die zweimalige Erwähnung der Dämmerung und auch der Übergang zum Schattenreich der Nacht glauben machen könnte. Es ist vielmehr der poetische Aufbruch in eine radikal behauptete Freiheit lyrischen Ausdrucks, welche die eigene Körperlichkeit zum Schauplatz einer singulär dezentrierten Subjektivität macht. In César Vallejos Gedicht XIII in Trilce ist es eine den Menschen transzendierende Gewalt, die Gewalt auch und gerade über Körper und Körperlichkeit erhält.

Dieses Gedicht besitzt zweifellos eine narrative Grundstruktur, die nicht zuletzt anhand der Verben sichtbar wird, dann aber zunehmend durch Interjektionen vorangetrieben zu sein scheint. Es führt auf sehr sorgfältige Weise die langsame und stetige Verabschiedung von Intellekt und Bewusstsein vor: zunächst durch das „simplificar“, dann durch das Absterben eines alten Gefühls, schließlich die Verabschiedung der „conciencia“ und das Aufgehen in der alles mit sich fortreißenden Lust. Der Schrei, der mit dem letzten Lexem dieses Gedicht enden lässt, gehört nicht mehr der spanischen Sprache an. Dafür aber einer anderen?

Die deutsche Sprache hätte für die letzte Gedichtzeile einige Lexeme anzubieten. Versuchen wir es einmal auf spielerische Weise. Das „Odu“ ließe sich leicht als romantischer Ausruf des liebenden Subjekts identifizieren, tausendfach verbürgt in einschlägigen Gedichten der Romantik. Wenn wir die Lexemgrenze so setzen, dann beträfe die nächste das „mod“, was auf ungeklärte Weise die Moderne, das Moderne oder vielleicht auch das Modische oder die Mode in das Gedicht hineinblendet. Denken wir an Charles Baudelaires Wort „Il faut être absolument moderne“, das vom Dichter eine absolute Modernität all seines Tuns und Denkens einfordert. Auf derselben Ebene siedelt sich das nachfolgende Lexem an, das gleichsam die Wirkung von „mod“ verstärkt: „neu“ oder vielleicht auch „neur“, also neuer. Es scheint um Innovation bis zur Grenze des noch Sagbaren zu gehen: „tse“. Sie merken, welche Spielmöglichkeiten auch dieser letzte Ausruf in Trilce offenlässt und wie diese Gedichte nicht ohne eine aktive, kreative, intervenierende Leserschaft auskommen können. Oh du moderne neue Welt der Lyrik, des Unsagbaren!

Aber Sie haben, vielleicht auch durch meine deutsche Übersetzung, längst bemerkt, worum es hier geht: Denn César Vallejo hat an dieser Schlussstelle seines Gedichts ein Palindrom eingesetzt, um die orgiastische Pointe gleichsam im Unverständlichen auf die Spitze, auf einen dem Akt im Gedicht entsprechenden Höhepunkt zu treiben. Im Palindrom wird der vorausgehende Vers Buchstabe für Buchstabe umgedreht und in einer wiederum linearen Sequenz angeordnet. Sie können sich bei meinem Nachnamen sehr gut vorstellen, dass mir Palindrome 
sehr lieb sind und ich deren Verwendung in der barocken wie der zeitgenössischen Lyrik sehr schätze.

Wir könnten das Bild der Frau, das bei Vallejo in Bezug auf die Vagina stets mit der Vorstellung von Vergehen, Grab und Tod besetzt ist, vergleichen mit dem Bild des weiblichen Körpers bei Lyrikerinnen Hispanoamerikas im selben Zeitraum, bei Autorinnen wie Gabriela Mistral, Juana de Ibarbourou oder Alfonsina Storni. Wir werden dies zu einem späteren Zeitpunkt in unserer Vorlesung auch tun und den männlichen Blick mit weiblichen Blicken nicht zuletzt auch auf männliche Körper konfrontieren. Doch möchte ich an dieser Stelle - um unser Bild der Sprachgewalt César Vallejos abzurunden - noch einige weitere Überlegungen zu diesem großen peruanischen Avantgardisten nachschieben, der zu allen Zeiten - und nicht nur in den Zeiten der historischen Avantgarde - sicherlich ein Avantgardist gewesen wäre!

Nach Trilce im Jahre 1922 veröffentlichte César Vallejo, von wenigen lyrischen Texten in Zeitschriften abgesehen, so gut wie keine Gedichte mehr. Vallejo ist freilich kein Fall für die von Enrique Vila-Matas so bezeichnete „Literatura del No“: Er beschloss nicht wie ein Arthur Rimbaud, von einem Tage auf den anderen zu verstummen. Aber es wurde doch deutlich stiller um ihn. Zwar scheint er noch vor seinem Tod wieder daran gedacht zu haben, einen oder mehrere Gedichtbände zu veröffentlichen; doch überraschte ihn der Tod 1938 in Paris, noch bevor er derartige Pläne oder Vorhaben umsetzen konnte.

So fand man 1938 neben vielen anderen Gedichten, welche später unter dem Titel Poemas humanos erscheinen sollten, einen Gedichtband mit dem Titel España, aparta de mí este cáliz, der die Erfahrungen Vallejos im Spanischen Bürgerkrieg reflektierte und besang. Schon früher hatte Vallejo bemerkt, dass er mit Trilce an eine Grenze des Sagens vorgestoßen war, die nicht leicht hätte weiter ausgedehnt oder avantgardistisch vorgeschoben werden können. Dies bedeutet nicht, dass Vallejo etwa in eine avantgardistische oder allgemein schöpferische Sackgasse gelangt wäre, nein! Aber nun standen andere Dinge im Vordergrund, die ihrerseits wiederum sein lyrisches Schreiben prägen sollten.

Seine avantgardistischen Aktivitäten widmeten sich in diesen Jahren dem Kampf um Spanien, dem Kampf um die Spanische Republik, der die Menschen und insbesondere die Intellektuellen der Zwischenkriegszeit bewegen und polarisieren sollte. César Vallejo ergriff in diesem Kampf Partei, selbstverständlich auf Seiten der Unterdrückten, der Arbeiter, der republikanischen Institutionen und Strukturen, die er trotz aller Fehler im Lager der Republik mit allem, was er hatte, verteidigte. Ich habe seine Teilnahme an den großen antifaschistischen Kongressen und Zusammenkünften bereits erwähnt; und als Dichter besang er die Mutter Spanien, die nicht untergehen dürfe: „Niños del mundo, / si cae España“ „Kinder der Welt, / Wenn Spanien fällt““. 
Der Peruaner Vallejo projizierte seine ganzen Ängste, aber auch all seine Kraft und seine politischen Hoffnungen auf das republikanische Spanien, das er aus militanter kommunistischer beziehungsweise sozialistischer Perspektive repräsentierte und darstellte. Er schilderte Spaniens Leiden, die „pobres“ der Spanischen Republik, Spaniens Milizionäre, den Kampf der Republikaner gegen die Kräfte des Faschismus, den immer verzweifelter werdenden Bürgerkrieg. Vor allem aber besang er Spaniens einfache Menschen, das spanische Volk, das dem Vorrücken des europäischen Faschismus immer schutzloser preisgegeben war.

Ich möchte Ihnen zum Abschluss unserer Beschäftigung mit César Vallejo ein Beispiel für diese Lyrik des peruanischen Dichters zeigen, diesmal freilich nicht das Titelgedicht dieses Bandes, sondern vielmehr das Gedicht Nummer XII, das zugleich den Titel Masa trägt:

Am Ende der Schlacht, und der Kämpfer schon tot, kam zu ihm ein Mensch und sagte ihm: „Stirb nicht, ich liebe Dich so sehr!“ Doch der Kadaver, ach!, starb weiter.

Es kamen zweie zu ihm und wiederholten: „Verlass uns nicht! Nur Mut! Zurück ins Leben!“ Doch der Kadaver, ach!, starb weiter.

Es kamen zwanzig, hundert, tausend, fünfhunderttausend und riefen: „Soviel Liebe, und nichts auszurichten gegen den Tod!“ Doch der Kadaver, ach!, starb weiter.

Es umstanden ihn Millionen von Individuen mit einem gemeinsamen Flehen: „Bleib, Bruder!“ Doch der Kadaver, ach!, starb weiter.

Sodann umstanden ihn alle Menschen auf der Erde; es sah sie der Kadaver, traurig, tief bewegt; er erhob sich langsam, umarmte den ersten Menschen; und begann zu gehen .... ${ }^{11}$

In diesem Gedicht César Vallejos, das den Menschen ins Zentrum rückt, scheint eine utopische Hoffnung auf, die immer das gesamte lyrische Werk des Dichters durchzog: die Vereinigung mit allen Menschen zu einem Einzigen, zu einer Einheit, zu einem Ganzen. So kann das Oxymoron des sterbenden Leichnams, des

11 Vallejo, César: Masa (aus España, aparta de mí este cáliz). In ders.: Obra poética completa. Introducción de Américo Ferrari. Madrid: Allianza Editorial 1983, S. 300. 
toten und doch nicht toten Kadavers, auch nicht gerettet werden, solange es nur Teile der Menschheit sind, wie groß sie auch immer sein mögen, die den Toten wieder zum Leben erwecken wollen.

Vergessen wir nicht, Es handelt sich um die Szenerie einer Schlacht, also der prototypischen Ausformung des bewaffneten Kampfes unter den Menschen, für den der Spanische Bürgerkrieg geradezu ein traumatisches Grundmuster lieferte, das nicht nur die Symbolik der „Dos Españas“, sondern auch zweier gegeneinander kämpfender Teile der Menschheit heraufbeschwor! Vallejo konnte noch nicht ahnen, dass dieser blutige Krieg um die Spanische Republik nur das Vorspiel war für einen weiteren Weltkrieg, der die Schrecken des Ersten Weltkriegs noch weit übertreffen sollte.

Doch gerade in dieser Situation eines politischen und militärischen, aber auch ideologischen Bürgerkrieges sowie einer klaren radikalen Parteinahme Vallejos beschwört das Gedicht die Einheit der Menschen, da nur so die Liebe gegen den Tod eine Chance haben könne. Der „primer hombre“, also „le premier homme“ (mit Albert Camus zu sprechen), umarmt den wieder in die Gemeinschaft zurückgekehrten Leichnam und begrüßt ihn liebevoll in der neuen Gemeinschaft aller Menschen auf der Erde. Dies ist die wahre Vereinigung, für die exemplarisch zunächst die Mutter mit ihrem Kind und später die körperliche Vereinigung der Liebenden gestanden hatten, für die nun aber die „Madre Patria“ Spanien, die neue kollektive Mutter, einstand. Sie alleine genügte jedoch nicht, da nur die universale Umarmung aller Menschen eine Überwindung jener existentiellen Zerrissenheit ermöglichen könnte, unter welcher Vallejo ein Leben lang litt und aus welcher seine Gedichte, seine zutiefst menschlichen Gedichte, entstanden.

Wir sollten uns hüten, diese Entwicklung, die sehr wohl widersprüchlich war und keineswegs im Sozialismus oder Kommunismus aufging, ideologisch auszuschlachten, wie dies so oft in den Debatten um den peruanischen Dichter geschehen ist. Wir sollten jedoch zur Kenntnis nehmen, dass César Vallejo wie vielleicht kein anderer Lyriker seiner Zeit die tragische Verknüpfung literarischer und politischer Avantgarde in seinem Schaffen wie in seinem Werk vorführte und somit eine existentielle Verankerung seiner avantgardistischen Dichtkunst schuf, wie sie wohl keinem anderen hispanoamerikanischen Poeten jener Epoche gelang. Vielleicht liegt hierin die tiefe Bedeutung von Vallejos Schaffen. 